\title{
Spontaneous Regression of a Cervical Intraspinal Cyst in a Dog
}

\author{
Hiroaki KAMISHINA ${ }^{1,2)}$, Hiroya OGAWA ${ }^{3)}$, Masaaki KATAYAMA ${ }^{4)}$, Jun YASUDA ${ }^{1)}$, Reeko SATO ${ }^{1)}$ and \\ Koujiro TOHYAMA ${ }^{2)}$
}

${ }^{1)}$ Divisions of Small Animal Internal Medicine and ${ }^{4}$ Small Animal Surgery, Department of Veterinary Medicine, Faculty of Agriculture, Iwate University, 3-18-8 Ueda, Morioka, Iwate 020-8550, ${ }^{2)}$ Center for EM and Bio-Imaging Research, Laboratory for NanoNeuroanatomy, Iwate Medical University, 19-1 Uchimaru, Morioka, Iwate 020-8505 and ${ }^{33}$ Tohno Animal Clinic, 17-78-3 Shiraiwa, Matsuzaki, Tohno, Iwate 028-0541, Japan

(Received 26 August 2009/Accepted 24 October 2009/Published online in J-STAGE 1 December 2009)

ABSTRACT. We report a cervical intraspinal cyst in a dog that was initially tetraparetic but spontaneously recovered completely. MRI revealed a well-demarcated intraspinal cyst located dorsally to a degenerated intervertebral disc. The location of the cyst and its signal features on MRI resembled those of discal cysts previously reported in humans. It has been reported in dogs that clinical signs of a intraspinal cyst are similar to those of intervertebral disc herniation and both conditions require surgical intervention. Unexpectedly, our case showed rapid spontaneous recovery and the follow-up MRI revealed complete resolution of the intraspinal cyst and spinal cord compression. Spontaneous recovery of degenerative intraspinal cyst may occur in dogs, similar to rare human cases as reported previously. KEY WORDS: canine, intervertebral disc disease, intraspinal cyst, magnetic resonance imaging.

J. Vet. Med. Sci. 72(3): 349-352, 2010

Most cervical intervertebral disc diseases in dogs are a result of disc extrusion rather than disc protrusion and therefore cause acute onset of clinical signs. Frequently observed clinical signs include paraspinal hyperesthesia, tetraparesis, and tetraplegia. Similar clinical signs can be observed in dogs with various intraspinal cysts although in these cases the clinical onset may be more slowly progressive $[5,12]$. Intraspinal cyst is a collective term used to describe intraspinal cyst formation originating from the synovium of the articular process (e.g. synovial cyst) [5], periarticular fibrous tissue of the intervertebral joints (e.g. ganglion cyst) [15], or an ectodermal tissue (e.g. epidermoid cyst) [13]. Dilation of the subarachnoid space has been termed subarachnoid cyst although the lack of epithelial cell lining suggests these are not true cysts [6]. Degenerated intervertebral disc could be another origin of intraspinal cysts which have been termed discal cyst and reported in humans [10] and dogs [9]. In this report, we present a case with a presumptive diagnosis of discal cyst based on MRI findings. Unique findings were that this case fully recovered spontaneously and the cyst disappeared at the follow-up MRI.

A six-year-old, neutered female Border Collie presented to Iwate University, Veterinary Teaching Hospital for evaluation of acute onset of non-ambulatory tetraparesis that had occurred one day ago. The dog did not receive treatment prior to referral. On presentation, the dog appeared alert and showed no signs of pain or discomfort. On neurological examination, postural reactions were reduced in all limbs with right side being slightly worse. Spinal reflexes were present in all limbs and the cutaneous trunci reflex was nor-

\footnotetext{
* Correspondence to: Kamishina, H., Division of Small Animal Internal Medicine, Department of Veterinary Medicine, Faculty of Agriculture, Iwate University, 3-18-8 Ueda, Morioka, Iwate 020-8550, Japan.

e-mail:kamicna@iwate-u.ac.jp
}

mal. There were no signs of cranial nerve deficits and hyperpathia along the entire spine. Neuroanatomical localization was C1-C5 spinal cord segment. Results of complete blood count and serum biochemistry were unremarkable. Plane radiographs of the cervical spine were also unremarkable.

Two days later, the dog presented for magnetic resonance imaging (MRI) at which point the dog was severely ataxic but ambulatory. MRI of the cervical spine was performed using a 3.0 T Signa VH-I (GE, Milwaukee, WI, U.S.A.) at the Highfield MRI Research Institute, Iwate Medical University. The dog was premedicated with butorphanol and midazolam intravenously. Anesthesia was induced with intravenous propofol and maintained with isoflurane/oxygen mixture. T2-weighted, pre- and post-contrast T1weighted with fat-suppression, and FLAIR images were obtained in sagittal and transverse planes. The contrast medium used was Omniscan (GE Health Care, Giles, U. K.). MRI revealed mild disc protrusion at $\mathrm{C} 3 / 4$ and $\mathrm{C} 4 / 5$, degeneration of the nucleus pulposus of $\mathrm{C} 4 / 5 \mathrm{disc}$, and an intraspinal cyst caudal to the herniated $\mathrm{C} 3 / 4$ disc (Fig 1a). Bulging of the annulus fibrosus and intraspinal cyst were seen on T1weighted images (Fig. 1b). On transverse images, there was a well-demarcated cyst located in the ventral spinal canal between the laterally located epidural venous plexus at the C3/4 intervertebral disc level. The cyst appeared somewhat heterogeneous and hyper- to iso-intense to the cerebrospinal fluid (CSF) on T2-weighted sequences (Fig. 2a). The spinal cord grey matter was deviated dorsally and slightly laterally which suggested the presence of extradural compression of the spinal cord by the cyst (Fig. 2a). The cyst appeared hypo-intense to almost signal void on T1-weighted sequences in comparison to the spinal cord parenchyma (Fig. 2b). The signal intensity of the cyst was reduced and appeared iso-intense to the surrounding parenchyma on 

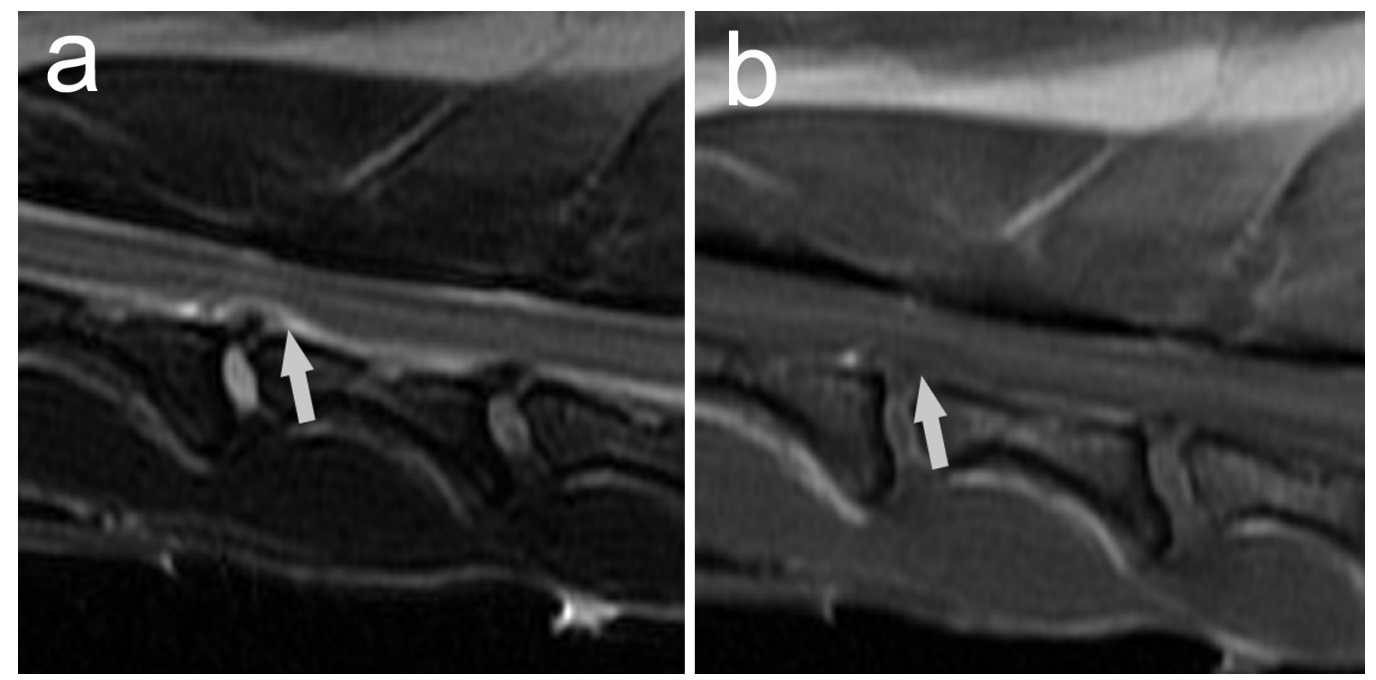

Fig. 1. Sagittal T2- (a) and T1- (b) weighted magnetic resonance images of the upper cervical spine. C3-4 and C4-5 intervertebral discs show herniation. Caudal to the herniated $\mathrm{C} 3-4$ disc a discal cyst (arrow) appears iso-intense to the cerebrospinal fluid (CSF). C4-5 intervertebral disc shows slight signal decrease.
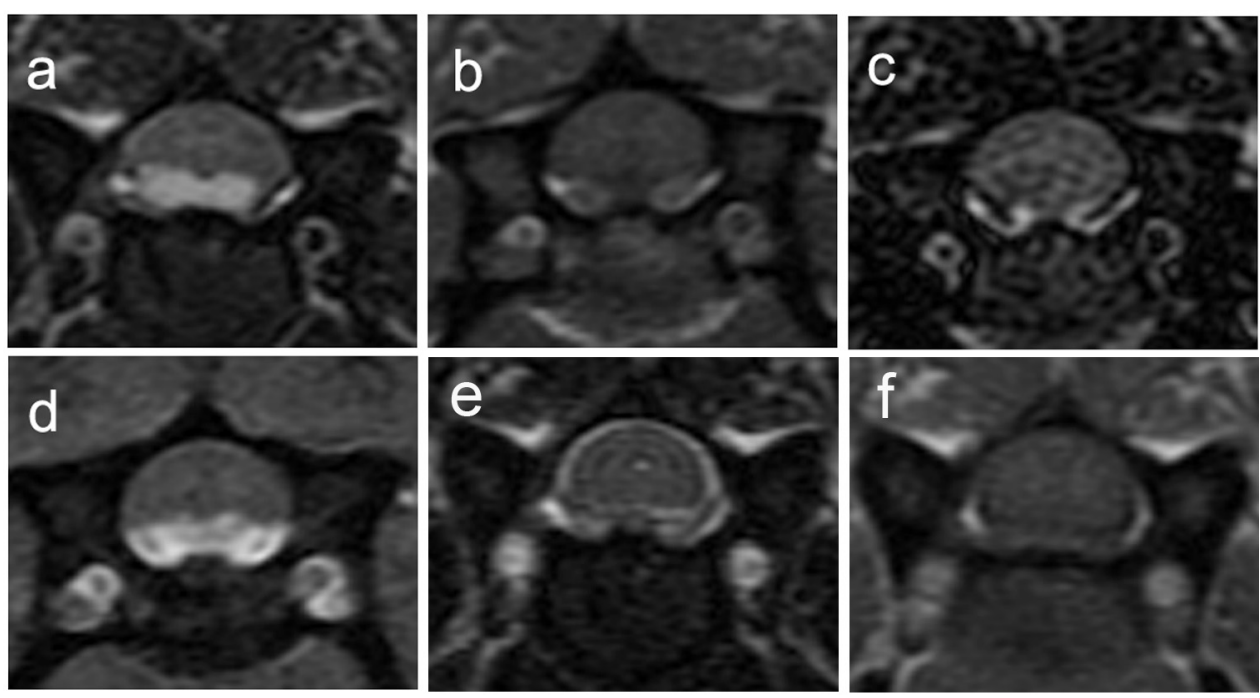

Fig. 2. Transverse images of the spine at the C3-4 intervertebral disc level. (a) On T2-weighted image a peanut-shape cyst located between the epidural venous plexus is compressing the ventral portion of the spinal cord. Note dorsal deviation of the grey matter, suggesting the presence of extradural compression. The cyst is hyper- to iso-intense to the CSF. (b) On T1-weighted image, the cyst appears hypo-intense to the spinal cord parenchyma. (c) On FLAIR image, the signal of the cyst is suppressed. (d) After administration of contrast medium, the cyst shows clear rim enhancement. (e), (d) Follow-up MRI study performed 44 days after the clinical onset revealed complete absorption of the cyst and relief of spinal compression on T2- (e) and T1-weighted (f) images.

FLAIR sequences (Fig. 2c). In contrast study, the cyst showed clear peripheral enhancement (Fig 2d).

The MRI features suggested the presence of an intraspinal cyst, possibility a discal cyst, because of its anatomical relationship to the intervertebral disc and characteristic signal intensities on each sequence. Clinical symptoms improved dramatically with only physical therapy over the first 10 days and the dog completely recovered after 2 weeks. Follow-up MRI study performed 44 days after the clinical onset revealed complete absorption of the cyst and relief of spinal compression (Fig. 2e and f). Signal intensity of the nucleus pulposus of $\mathrm{C} 3 / 4$ decreased compared to that seen on the first MRI, indicating progression of disc degeneration. The neurological status of the dog remains normal 10 months later.

There are several types of intraspinal cysts reported in 
dogs, including arachnoid cysts, synovial cysts, dermoid cysts, and ganglion cysts $[5,6,13,15]$. Of different types of intraspinal cysts, those having connection to the corresponding intervertebral disc have been termed discal cysts, which is a relatively new clinical entity in humans. [10] There is only 1 report in dogs with similar conditions, describing MRI features of discal cysts in 7 dogs [9]. In their study, all dogs recovered their ambulatory function following surgical resection of the cyst and spinal cord decompression. Our case recovered spontaneously and therefore surgery was not performed. Although definitive diagnosis could not be made without pathological confirmation we speculate that the intraspinal cyst seen in our case had communication with the herniated disc based on its anatomical relation to the degenerated C3/4 disc and characteristic MRI features. Pathologically, discal cysts are composed of a thin connective tissue that lacks inner lining cells and serous or serosanguinous contents $[2,8]$. Although the exact pathogenesis of discal cysts remains unclear presence of disc material in the cyst and occasional hemosiderin deposits in the cyst wall support the contention that herniation of a degenerated disc and subsequent hemorrhage from the epidural venous plexus may be the prime mechanisms of cyst formation [2, $7,14]$. Discal cysts produce extradural compression of the spinal cord or nerve root leading to clinical signs indistinguishable from those of intervertebral disc herniation in humans. In dogs, Konar et al. reported that the clinical signs of dogs with discal cysts were also similar to typical intervertebral disc herniation [9].

Definitive diagnosis of discal cysts can be made by discography or CT discography to prove communication of the cyst and the corresponding intervertebral disc $[2,14]$. Clinically, however, MRI has been the most effective diagnostic modality. Lee et al. reported detailed MRI features of lumbar discal cysts in 9 human cases [11]. They reported that lumbar discal cysts were located in the ventrolateral epidural space of the spinal canal with occasional extension into the lateral recess and showed rim enhancement on contrast study [11]. These MRI features were similar in canine cases reported by Konar et al. [9]. Whereas the number of human cases reports the lumbar vertebral area as the most frequent site of discal cyst formation the lesion in our case was located in the cervical spine. Interestingly, 6 of 7 canine cases in Konar's study had discal cysts in the cervical area, implying possible biomechanical and/or nutritional differences of the intervertebral discs in humans and dogs.

Since clinical signs of discal cysts are caused by spinal or nerve root compression discal cysts are treated surgically similar to herniated discs. Hemilaminectomy and cyst resection are effective in most reported human cases; however, recurrence does occur on rare occasions [11]. All canine cases in Konar's study were treated surgically and quickly recovered locomotor function. Our case unexpectedly recovered spontaneously with only physical therapy and completely regained locomotor function by 2 weeks after the onset. Although rare, spontaneous recovery of discal cysts has also been reported in humans $[3,4]$. In Demae- rel's report, a 44-year old man had a disc herniation and discal cyst at L1-L2 [4]. The patient's symptoms were spontaneously alleviated 3 weeks later and follow-up MRI 4 months later showed complete regression of the lesion. Given that spontaneous regression of a herniated intervertebral disc and a synovial cyst has been reported [1] spontaneous recovery of discal cyst may occur by a similar process.

We report a spontaneously recovered cervical intraspinal cyst in a dog. A high field MRI unit was very effective in diagnosing the cyst and findings were similar to discal cysts previously reported in humans and dogs. Spontaneous recovery may occur in a cervical discal cyst in dogs like rare cases in humans. These observations add to our current knowledge about this newly recognized entity in dogs.

ACKNOWLEDGMENTS. We thank Prof. Makoto Sasaki for his helpful critique of the manuscript and Mr. Yutaka Matsumura for his excellent technical assistance in MRI.

\section{REFERENCES}

1. Chang, H., Park, J. B. and Kim, K. W. 2000. Synovial cyst of the transverse ligament of the atlas in a patient with os odontoideum and atlantoaxial instability. Spine 25: 741-744.

2. Chiba, K., Toyama, Y., Matsumoto, M., Maruiwa, H., Watanabe, M. and Nishizawa, T. 2001. Intraspinal cyst communicating with the intervertebral disc in the lumbar spine: discal cyst. Spine 26: 2112-2118.

3. Chou, D., Smith, J. S. and Chin, C. T. 2007. Spontaneous regression of a discal cyst. Case report. J. Neurosurg. Spine 6: 81-84.

4. Demaerel, P., Eerens, I., Goffin, J. and Wilms, G. 2001. Spontaneous regression of an intraspinal disc cyst. Eur. Radiol. 11: 2317-2318.

5. Dickinson, P. J., Sturges, B. K., Berry, W. L., Vernau, K. M., Koblik, P. D. and Lecouteur, R. A. 2001. Extradural spinal synovial cysts in nine dogs. J. Small Anim. Pract. 42: 502-509.

6. Gnirs, K., Ruel, Y., Blot, S., Begon, D., Rault, D., Delisle, F., Boulouha, L., Colle, M. A., Carozzo, C. and Moissonnier, P. 2003. Spinal subarachnoid cysts in 13 dogs. Vet. Radiol. Ultrasound. 44: 402-408.

7. Hwang, J. H., Park, I. S., Kang, D. H. and Jung, J. M. 2008. Discal cyst of the lumbar spine. J. Korean Neurosurg. Soc. 44: 262-264.

8. Koga, H., Yone, K., Yamamoto, T. and Komiya, S. 2003. Percutaneous CT-guided puncture and steroid injection for the treatment of lumbar discal cyst: a case report. Spine 28: E212216.

9. Konar, M., Lang, J., Fluhmann, G. and Forterre, F. 2008. Ventral intraspinal cysts associated with the intervertebral disc: magnetic resonance imaging observations in seven dogs. Vet. Surg. 37: 94-101.

10. Kono, K., Nakamura, H., Inoue, Y., Okamura, T., Shakudo, M. and Yamada, R. 1999. Intraspinal extradural cysts communicating with adjacent herniated disks: imaging characteristics and possible pathogenesis. AJNR Am. J. Neuroradiol. 20: 1373-1377.

11. Lee, H. K., Lee, D. H., Choi, C. G., Kim, S. J., Suh, D. C., Kahng, S. K., Roh, S. W. and Rhim, S. C. 2006. Discal cyst of the lumbar spine: MR imaging features. Clin. Imaging. 30: 326-330. 
12. Skeen, T. M., Olby, N. J., Munana, K. R. and Sharp, N. J. 2003. Spinal arachnoid cysts in 17 dogs. J. Am. Anim. Hosp. Assoc. 39: 271-282.

13. Steinberg, T., Matiasek, K., Bruhschwein, A. and Fischer, A. 2007. Imaging diagnosis--intracranial epidermoid cyst in a Doberman Pinscher. Vet. Radiol. Ultrasound. 48: 250-253.

14. Tokunaga, M., Aizawa, T., Hyodo, H., Sasaki, H., Tanaka, Y. and Sato, T. 2006. Lumbar discal cyst followed by intervertebral disc herniation: MRI findings of two cases. J. Orthop. Sci. 11: $81-84$.

15. Webb, A. A., Pharr, J. W., Lew, L. J. and Tryon, K. A. 2001. MR imaging findings in a dog with lumbar ganglion cysts. Vet. Radiol. Ultrasound. 42: 9-13. 